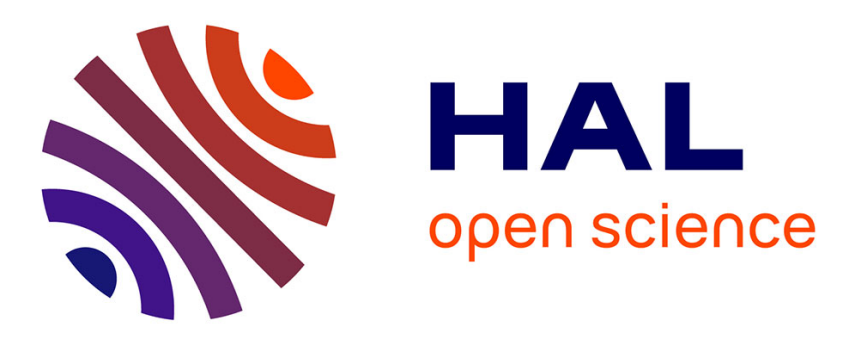

\title{
Stabilization and controlled association of superparamagnetic nanoparticles using block copolymers
}

Bruno Frka-Petesic, Jérôme Fresnais, Jean-François Berret, Vincent Dupuis, Régine Perzynski, Olivier Sandre

\section{- To cite this version:}

Bruno Frka-Petesic, Jérôme Fresnais, Jean-François Berret, Vincent Dupuis, Régine Perzynski, et al.. Stabilization and controlled association of superparamagnetic nanoparticles using block copolymers. The 3rd Moscow International Symposium on Magnetism (MISM), Jun 2008, Moscou, Russia. pp.667670, 10.1016/j.jmmm.2008.11.021 . hal-00346968

\section{HAL Id: hal-00346968 https://hal.science/hal-00346968}

Submitted on 31 May 2020

HAL is a multi-disciplinary open access archive for the deposit and dissemination of scientific research documents, whether they are published or not. The documents may come from teaching and research institutions in France or abroad, or from public or private research centers.
L'archive ouverte pluridisciplinaire HAL, est destinée au dépôt et à la diffusion de documents scientifiques de niveau recherche, publiés ou non, émanant des établissements d'enseignement et de recherche français ou étrangers, des laboratoires publics ou privés. 


\title{
Stabilization and controlled association of superparamagnetic nanoparticles using block copolymers
}

\author{
Bruno Frka-Petesic ${ }^{\mathrm{a}}$, Jérôme Fresnais ${ }^{\mathrm{b}}$, Jean-François Berret ${ }^{\mathrm{b}}$, Vincent Dupuis ${ }^{\mathrm{a}}$, \\ Régine Perzynski ${ }^{\mathrm{a}}$ and O. Sandre \\ ${ }^{a}$ LI2C, UMR 7612 CNRS Université Pierre et Marie Curie Paris-VI, case 63, 4 place Jussieu, F-75252 Paris Cedex 05 France \\ ${ }^{b}$ Lab. MSC, UMR 7057 Bâtiment Condorcet 10 rue Alice Domon et Léonie Duquet, F-75205 Paris Cedex 13 France
}

Article available online: 27 November 2008

\begin{abstract}
Mixing in aqueous solutions polyelectrolyte-neutral block copolymers with oppositely charged species, spontaneously forms stable core-shell complexes, which are electrostatically driven. We report here on the structural and orientational properties of such mixed magnetic nanoclusters made of magnetic iron oxide nanoparticles (MNP) and polyelectrolyte-neutral block copolymers. Small Angle Neutron Scattering and Transmission Electron Microscopy experiments allows to probe the innercore nanoparticle organization, leading to an average inter-particle distance and confirming the hierarchical internal structure of the clusters. Thanks to the MNP optical anisotropy, we also probe the under-magnetic field orientational properties of the core-shell clusters and their dynamical rotational relaxation.

Author manuscript version of article available in its final form on the publisher's website: DOI 10.1016/j.jmmm.2008.11.021
\end{abstract}

Pacs : 82.70.Dd, 81.07.-b, 83.80.Uv, 75.50.Mm, 81.16.Dn

Keywords: Colloids, Nano-composites, Block copolymers, Magnetic liquids, Self-assembly

\section{Introduction}

Building up hybrid organic/inorganic nano- and meso-clusters of controlled size and shape [1-2] is an important issue for many applications. In [3-4] Boal et al have suggested to use inorganic nanoparticles as building blocks combined together to synthesize colloidal or supracolloidal assemblies in a polymer- mediated "Bricks and Mortar" approach, to take benefit of the nanoparticle properties and to control as well the stability of complexes. Indeed, inorganic nanoparticles are emerging as central constituents for future nanotechnological developments spreading over a wide variety of material science applications, from catalysis and opto-electronics to biosciences.

Some core-shell structures have already been investigated using polyelectrolyte-neutral copolymer as a mortar and either oppositely charged surfactants [5] or nanoparticules as "bricks" [6-8]. The core is

* Corresponding author. Tel.: +33-1-4427-3267; fax: +33-1-4427-3228

E-mail address: regine.perzynski@upmc.fr. 
then described as a complex coacervation microphase made of "bricks" connected with the polyelectrolyte blocks, while the shell is made of neutral chains organized in a dense brush corona that insures the stability of the whole aggregate. Employed as such fundamental bricks, magnetic nanoparticles (MNPs) made of iron oxides drive particular interest in material science and biomedicine as MRI contrast agents [6], useful for magnetic cell labelling [9] or hyperthermia [10]. We study here the structural and orientational properties of such MNP hybrid clusters .

\section{Synthesis of the co-assembly}

The MNP's used in this work to built up the particle/polymer clusters are negatively charged $\gamma$ $\mathrm{Fe}_{2} \mathrm{O}_{3}$ maghemite nanoparticles, chemically synthesized in LI2C following the Massart's protocol for ionic ferrofluid (FF) synthesis. It consists in a polycondensation of iron salts in alkaline aqueous medium [11]. The obtained nanoparticles can be stabilized in strong acidic conditions $(\mathrm{pH}<2)$ thanks to their positive structural surface charges. To obtain a stable colloidal FF dispersion at neutral $\mathrm{pH}$, the MNPs are then coated with citrate ions, thanks to an adsorption equilibrium. Zetametric measurements show that the MNP's bear a strong and negative surface charge with a Zeta potential of $-30 \mathrm{mV}$. The typical charge density of these MNP's is 1.7 electronic charges per $\mathrm{nm}^{2}$ at a concentration of free citrate species $\left[\mathrm{Na}_{3} \mathrm{Cit}\right]=10 \mathrm{mM}$ and $\mathrm{pH}=7$ [12]. The nanoparticles are magnetic monodomains with a saturation magnetization $\mathrm{m}_{\mathrm{S}}$ of the order of $2.510^{5}$ $\mathrm{A} / \mathrm{m}$. Their FF dispersions are superparamagnetic. Probed by vibrating sample magnetometry they allow determining the size distribution of the roughly spherical MNP's, which is obtained by an adjustment of the FF dispersion magnetization to a $1^{\text {st }}$ Langevin law weighted by a log-normal distribution of diameter. The distribution is characterized by a mean diameter $\mathrm{d}_{0}=\exp <\ln (\mathrm{d})>$ and a standard deviation $\sigma$ of $\ln (\mathrm{d})$ [13]. Two MNP's are used here (see table 1).

The polyelectrolyte-neutral di-block copolymer is a poly(trimethylammonium ethylacrylate methyl sulfate) $-b$ - poly(acrylamide) or PTEA- $b-$ PAM. It is synthesized by controlled radical polymerization in solution and is provided by Rhodia [14]. Fig. 1 gives the chemical formula of the two monomers.

\begin{tabular}{|c|c|c|}
\hline MNP Sample & do $(\mathrm{nm})$ & $\sigma$ \\
\hline MNP1 & 6.3 & 0.23 \\
\hline MNP2 & 8.5 & 0.29 \\
\hline
\end{tabular}

Table 1-MNP size characteristics.

In solution, the polyelectrolyte PTEA portion is positively charged whereas the PAM portion is neutral. We thus expect that positively charged PTEA blocks would adsorb on the surface of the negatively<smiles>CCC(CC)C(=O)OCC[N+](C)(C)C</smiles>

Fig. 1 - Chemical structure of the PTEA and PAM blocks entering in the composition of the co-polymers.

charged nanoparticles, and then make the nanoparticles aggregate into a dense core, while the neutral PAM blocks provide a steric repulsion limiting the cluster to a finite size and stabilizing it in the solution. Indeed the PAM supplies the clusters with a neutral corona of much lower density. The synthesis is performed by one-shot mixing at low concentration ( $1 \% \mathrm{wt}$ max.) of stock nanoparticle and co-polymer solutions using PTEA-PAM di-blocks of various molecular weights to produce clusters of various sizes and shapes. The relative amount of each species is monitored by the charge ratio, which is fixed to unity [6]. Three different molecular weights for PTEA- $b$-PAM block combinations are probed to synthesize MNP-copolymer nanoclusters: (A) $2 \mathrm{kD}$ $60 \mathrm{kD},(\mathrm{B}) 5 \mathrm{kD}-30 \mathrm{kD}$ and $(\mathrm{C}) 11 \mathrm{kD}-30 \mathrm{kD}$.

\section{Structural properties}

Small Angle Neutron Scattering, performed on PACE and PAXY spectrometers in LLB-CE Saclay- 
France, is first used to investigate the structural properties of the synthesized nanoclusters as a function of the scattering vector $\mathrm{q}$. As the clusters are dispersed in $\mathrm{H}_{2} \mathrm{O}$, the predominant contribution to the overall neutron scattering cross-section is expected to come from the nuclear scattering of the maghemite nanoparticles, that are constituting the cluster cores. Figure 2 presents the typical qdependent intensity scattered by a cluster dispersion

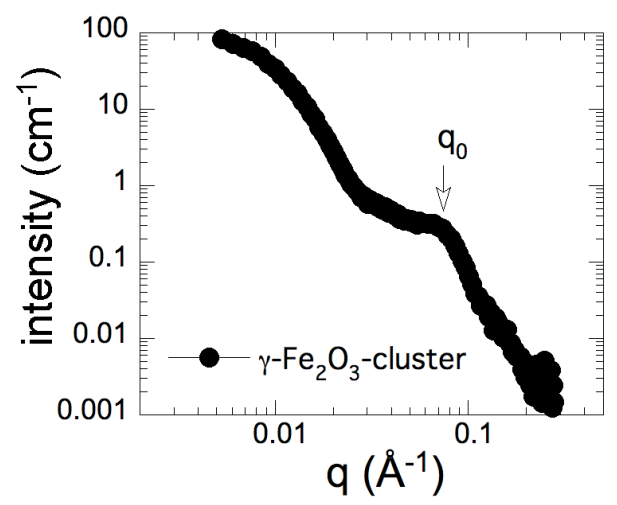

Fig. $2-\mathrm{q}$ dependence of the neutron intensity, scattered by a dispersion of MNP1/B-copolymer clusters

kere based on nanoparticles MNP1 and B-type copolymers). The spectra present three general scattering features : i) a strong forward scattering at low q's (related to the overall cluster shape), ii) a shoulder in the intermediate q-range (marked by an arrow at $\mathrm{q}_{0}$ in fig.2) and iii) a $\mathrm{q}^{-4}$ decrease of the intensity at high q's (to be associated to the MNP form factor in the Porod regime). Interpreting the wave vector $\mathrm{q}_{0}$ as the first order of the particleparticle structure factor leads to a mean interparticle distance inside the cluster of the order of $d_{i n t}=2 \pi / q_{0}$ $=78 \AA$, meaning a volume fraction $\Phi_{\text {int }}$ of magnetic particles inside the cluster core of the order of $27 \%$.

Some complementary transmission electron cryomicroscopy, performed in Centre de Recherches Aubervilliers-Rhodia-France, on the same dispersion as that of Figure 2 shows clusters with a dense internal organization of MNP (see Figure 3).

Here also the main contrast comes from MNPs, which appear distributed in separate clusters, well apart from each other, the invisible PAM corona preventing their agglomeration. If in Figure 3, the clusters appear polydisperse in size and in shape, they all present a similar internal structure. The densely

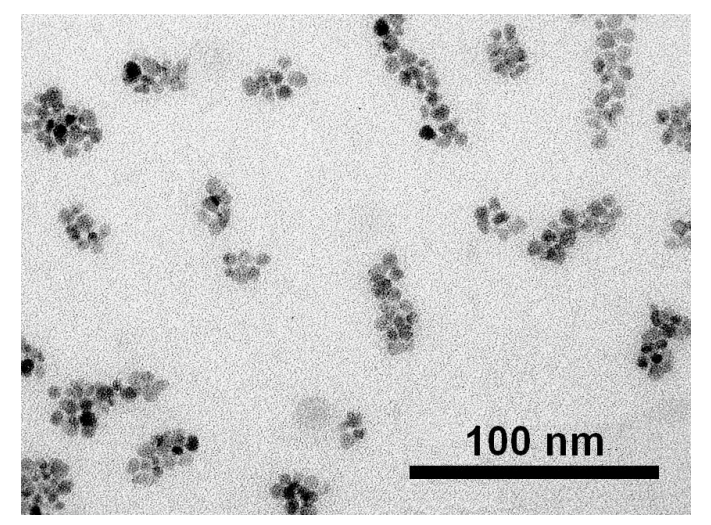

Fig. 3 - Cryo-TEM picture of a dispersion of MNP1/B-copolymer clusters

packed MNP remain all well apart from each other inside the clusters, being as if glued together by the PTEA chains. Figure 3 fairly agrees with an internal volume fraction of MNP's $\Phi_{\text {int }}$ of the order of $27 \%$ determined by SANS

Another series of SANS experiments performed with MNP2 nanoparticles and the whole copolymer

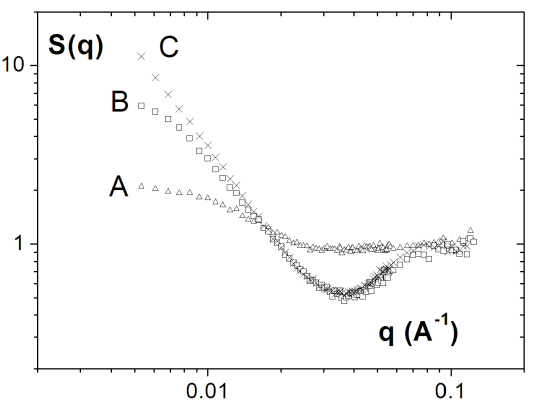

Fig 4 - Nanoparticle structure factor of cluster dispersions based on MNP2 nanoparticles and A, B or C copolymer.

series A, B and C lead to the cluster structure factors $\mathrm{S}(\mathrm{q})$ of Figure $4 . \mathrm{S}(\mathrm{q})$ is here obtained by dividing the intensity scattered by the cluster dispersion by that of the initial ferrofluid based on MNP2 nanoparticles alone (and renormalized by the respective volume fraction of NMP's in the two dispersions). The low q 
behavior of the three S(q) strongly depends on the copolymer used to build up the coacervates. With copolymer A, the clusters are small and contains only few nanoparticles (dimers on average with a gyration radius of the order of $80 \AA$, roughly twice that of the initial ferrofluid). With copolymers B and C, the cluster mean size grows too much to be precisely evaluated by such a scattering technique, the socalled Guinier regime being not reached at low q's.

Moreover as MNP2 nanoparticles are more polydisperse than MNP1, no bump at the interparticle distance inside the clusters is here visible. However, as it is also observed for NP1/B-copolymer clusters,

the structure factors of NP2/B- and NP2/C-

copolymer clusters present a marked correlation hole that can be associated to the hierarchical structure of the clusters. Indeed the coacervates have mean sizes larger than $100 \mathrm{~nm}$ and contain densely packed nanoparticles of much smaller size. The correlation hole in the cluster structure factor $\mathrm{S}(\mathrm{q})$ results from

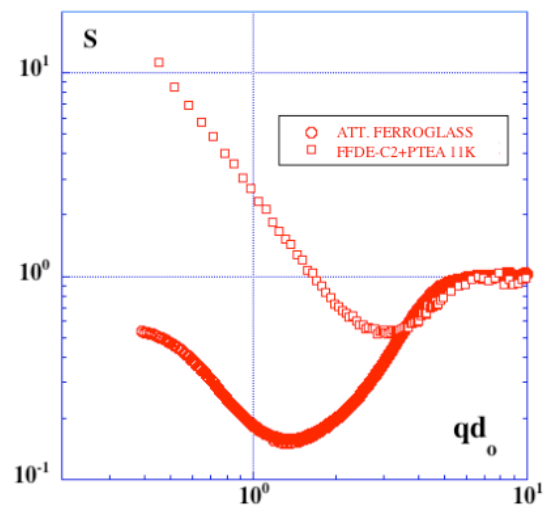

Fig. 5 - Comparison berween the structure factor of MNP2/Ccopolymer clusters (square symbols) and the structure factor of an attractive ferroglass at a nanoparticle volume fraction $\Phi=30 \%$ from [BrJP].

the competition between the cluster form factor (dominant at low q's) and the internal structure factor of the MNP inside the cluster (dominant at large q's).

To illustrate this point, Figure 5 compares the structure factor of a $30 \%$ attractive ferroglass based on nanoparticles of characteristics very close to these of MNP2 [15] with S(q) of NP2/C-copolymer clusters. If at low q's the cluster form factor rules up the cluster structure factor, at more local spatial scales (i.e. at large q's) the S(q) of the clusters clearly joins that of the ferroglass, comforting us with our previous $\Phi_{\text {int }}$ determination of the order of $27 \%$.

\section{Orientational properties}

The maghemite nanoparticles are optically anisotropic, their optical axis being linked to their magnetic moment through an anisotropy energy of the order of a few $10^{-21} \mathrm{~J}$. Under an applied field $\mathrm{H}$, dilute FF dispersions present an $\mathrm{H}$-dependent magneto-optical birefringence $\Delta \mathrm{n}$, which is directly linked to the statistical orientation of the particles by the field [16]. $\Delta \mathrm{n}$ is proportional to the volume fraction of nanoparticles and is well described by a $2^{\text {nd }}$ Langevin function, weighted by the particle size distribution.

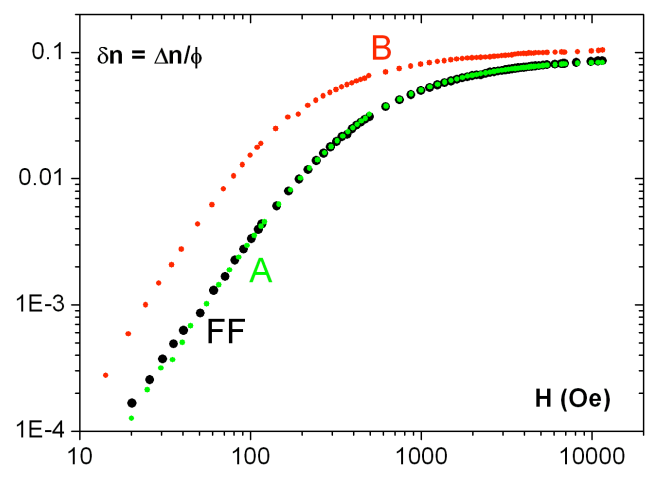

Fig6 - Static magneto-optical birefringence $\Delta \mathrm{n}$ normalized by the volume fraction $\Phi$ of nanoparticles in the dispersions. Lower curves : pure ferrofluid FF and dispersion of NP2/A-copolymer clusters; upper curve dispersion of NP2/B-copolymer clusters.

Dispersions of NP2/A- and NP2/B-copolymer clusters are optically probed under an applied magnetic field up to $\mathrm{H}=800 \mathrm{kA} / \mathrm{m}$. They remain stable under field (contrarily to dispersions of NP2/Ccopolymer clusters which destabilize). Figure 6 compares the field dependence of their magnetooptical birefringence to that of to the mother FF solution, in a reduce representation $\Delta \mathrm{n} / \Phi$ ( $\Phi$ being the total MNP volume fraction in the dispersions). If the birefringence of the dispersion of NP2/Acopolymer clusters remains very close to that of the pure ferrofluid FF, that of NP2/B-copolymer clusters largely differs. For this latter sample, an extra contribution from the shape orientation of the cluster 
structure is observed that should be addressed to a cluster shape anisotropy similar to that of the cryoTEM picture of Figure 3 .

A probing of the rotational diffusion of the clusters can be performed by analyzing the relaxation of magnetic birefringence after the application of a pulse of magnetic field of low amplitude $(5 \mathrm{kA} / \mathrm{m})$ [17]. Such relaxations are presented in Figure 7 for of NP2/A- and NP2/B-copolymer clusters. As it could

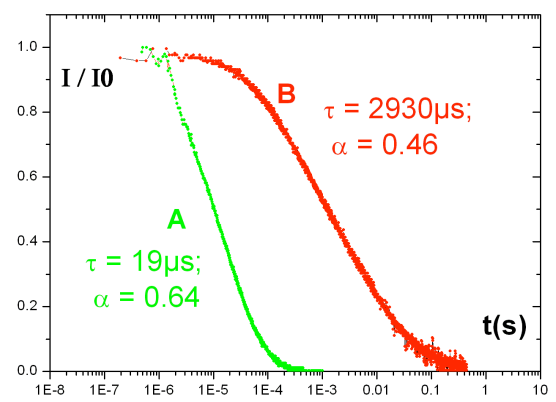

Fig7 - Relaxation of magneto-optical birefringence for dispersions of NP2/A-copolymer clusters and of NP2/B-copolymer ones.

be expected from cryo-TEM pictures, the magnetooptical relaxations of Figure 7 reveal very polydisperse distributions of rotational relaxation times that we analyse in terms of stretched exponentials (stretch exponent a given in Fig. 7). It leads to an hydrodynamic rotational diameter $d_{R}$ equal to $37 \pm 4 \mathrm{~nm}$ for NP2/A-copolymer clusters (shown to bear only two MNPs on average) and to $200 \pm 20 \mathrm{~nm}$ for the very anisotropic NP2/Bcopolymer clusters. These values are both coherent with SANS measurements and cryo-TEM pictures.

\section{Conclusions}

Coupled SANS, cryo-TEM and magnetic birefringence measurements give an insight on the internal structure of the magnetic hybrid coacervates successfully synthesized here by the electrostatic association of polyelectolyte-neutral copolymers and magnetic iron oxide nanoparticles. Inside the core, the magnetic nanoparticles are glued together by the polyelectolyte blocks with a nanoparticle density of the order of $27 \%$. The neutral branches of the diblock copolymers constitute the shell of much lower density. This corona provides the coacervates with colloidal stability in aqueous media. It is here probed hydrodynamically by the relaxation of magnetooptical birefringence, induced by applying a magnetic field on the dispersions of magnetic clusters.

\section{Acknowledgements}

We acknowledge support from Rhodia, from ANR "ITC-nanoprobe" and from ECO-NET program $\mathrm{n}^{\circ} 6274 \mathrm{QL}$. The authors thank A. Vacher and M. Airiau (C. R. Aubervilliers, Rhodia, France) for the cryo-TEM experiments and F. Cousin (LLB-CE Saclay- France) for his help in the SANS experiment.

\section{References}

[1] S. Lecommandoux, O. Sandre, F. Checot, R. RodriguezHernandez and R. Perzynski, Adv. Mat. 17 (2005) 712

[2] J. Fresnais, J.-F. Berret, B. Frka-Petesic, O.Sandre and R. Perzynski (submitted)

[3] A. K. Boal, F. Ilhan, J. E. DeRouchey, T. Thurn-Albrecht, T. P. Russell and V. M. Rotello, Nature 404, 746-748 (2000)

[4] A. K. Boal, B. L. Frankamp, O. Uzun, M. T. Tuominen, and V. M. Rotello, Chem. Mater. 16, 3252-3256 (2004)

[5] J.-F.Berret, P. Hervé, O. Aguerre-Chariol, J. Oberdisse, J. Phys. Chem B 2003, 107, 8111-8118

[6] K. Yokota, M. Morvan, J.-F. Berret and J. Oberdisse, Europhys. Lett. 69 (2005) 284-290.

[7] J.-F. Berret, N. Schonbeck, F. Gazeau, D. El Kharrat, O. Sandre, A. Vacher, and M. Airiau, J. Am. Chem. Soc. 128 (2006) $1755-1761$

[8] J.-F. Berret, A. Seghal, M. Morvan, O. Sandre, A. Vacher, and M. Airiau, J. Coll. Int. Sci. 303 (2006) 315-318

[9]C. Riviere, C. Wilhelm, F. Cousin, V. Dupuis, F. Gazeau and R. Perzynski, Eur. Phys. J. E 22 (2007) 1

[10]Q. A. Pankhurst, J. Connolly, S. K. Jones and J. Dobson, J. Phys. D. Appl. Phys. 36, R167-R181 (2003)

[11]R. Massart, IEEE Trans.Magn. MAG-17 (1981) 1247

[12]E. Dubois, V. Cabuil, F. Boué and R. Perzynski J. Chem. Phys 11 (1999) 7147

[13] J.-C. Bacri, R. Perzynski, S. Salin, V. Cabuil and R. Massart,

J. Magn. Magn. Mat. 62 (1986) 36

[14] D. Taton, A.-Z. Wilczewska and M. Destarac, Macromol. Rapid Commun. 2001, 22, 1497

[15] E. Wandersman, Yu. Chushkin, E. Dubois, V. Dupuis, G. Demouchy, A. Robert and R. Perzynski, Braz J Phys. (to appear in 2008)

[16] E. Hasmonay, E. Dubois, J.-C. Bacri, R. Perzynski and Yu. L. Raikher, Eur. J. Phys. B 5 (1998) 859

[17] C. Wilhelm, F. Gazeau, J. Roger, J.N. Pons, M.F. Salis, R. Perzynski and J.-C. Bacri, Phys. Rev. E 65 (2002) 031404 